\title{
Synaptic RTP801 contributes to motor-learning dysfunction in Huntington's disease
}

\author{
Núria Martín-Flores ${ }^{1,2}$, Leticia Pérez-Sisqués ${ }^{1,2}$, Jordi Creus-Muncunill ${ }^{1,2,3,4}$, Mercè Masana ${ }^{1,2,3,4}$, Sílvia Ginés (10) ${ }^{1,2,3,4}$, \\ Jordi Alberch (10) ${ }^{1,2,3,4}$, Esther Pérez-Navarro (10 ${ }^{1,2,3,4}$ and Cristina Malagelada (1),2,4
}

\begin{abstract}
RTP801/REDD1 is a stress-responsive protein that mediates mutant huntingtin (mhtt) toxicity in cellular models and is up regulated in Huntington's disease (HD) patients' putamen. Here, we investigated whether RTP801 is involved in motor impairment in HD by affecting striatal synaptic plasticity. To explore this hypothesis, ectopic mhtt was over expressed in cultured rat primary neurons. Moreover, the protein levels of RTP801 were assessed in homogenates and crude synaptic fractions from human postmortem HD brains and mouse models of HD. Finally, striatal RTP801 expression was knocked down with adeno-associated viral particles containing a shRNA in the R6/1 mouse model of HD and motor learning was then tested. Ectopic mhtt elevated RTP801 in synapses of cultured neurons. RTP801 was also up regulated in striatal synapses from HD patients and mouse models. Knocking down RTP801 in the R6/1 mouse striatum prevented motor-learning impairment. RTP801 silencing normalized the Ser473 Akt hyperphosphorylation by downregulating Rictor and it induced synaptic elevation of calcium permeable GluA1 subunit and TrkB receptor levels, suggesting an enhancement in synaptic plasticity. These results indicate that mhtt-induced RTP801 mediates motor dysfunction in a HD murine model, revealing a potential role in the human disease. These findings open a new therapeutic framework focused on the RTP801/Akt/mTOR axis.
\end{abstract}

\section{Introduction}

Huntington's disease (HD) is an autosomal-dominant neurodegenerative disorder caused by a CAG expansion in the exon-1 of the Huntingtin gene. This expansion encodes for a mutant form of the huntingtin (htt) protein that has been traditionally pointed out as responsible for the specific loss of medium-sized spiny neurons in the human striatum $^{1-3}$. However, the expanded CAG RNA was identified also as toxic and an active contributor to the HD pathogenesis ${ }^{4}$. HD manifests a triad of signs, including severe motor dysfunction with involuntary movements (chorea), cognitive impairment and neuropsychiatric

\footnotetext{
Correspondence: Núria Martín-Flores (nuria.martinf121@gmail.com) or Cristina Malagelada (cristina.malagelada@ub.edu)

${ }^{1}$ Department of Biomedicine, Faculty of Medicine, University of Barcelona, 08036 Barcelona, Catalonia, Spain

${ }^{2}$ Institut de Neurociències, University of Barcelona, 08036 Barcelona, Catalonia, Spain

Full list of author information is available at the end of the article

Edited by M. Agostini
}

symptoms. Even though mutant htt severely affects striatal neurons, other areas, such as cortex, hippocampus, amygdala or cerebellum, display synaptic alterations, atrophy, and/or neuronal death ${ }^{5,6}$.

Although neuronal death does not occur until late stages of HD, abnormal synaptic plasticity, and neuronal dysfunction are the main early pathogenic events that lead to neurodegeneration ${ }^{7-9}$. Owing to early synaptic dysfunction, observed both in the human and the mouse pathology, HD is considered a synaptopathy ${ }^{10-12}$. In this regard, one of the pathways that controls synaptic plasticity is the mechanistic target of rapamycin (mTOR) pathway, since it regulates translation and more notably, local protein synthesis at the spines ${ }^{13,14}$. Importantly, mTOR pathway is also involved in cytoskeleton remodeling to ensure proper formation and function of dendritic spines ${ }^{15}$.

mTOR kinase is the central component of mTOR complex (mTORC) 1 and 2. Both complexes share protein partners, but they have unique elements that define

\section{(c) The Author(s) 2020}

(c) (i) Open Access This article is licensed under a Creative Commons Attribution 4.0 International License, which permits use, sharing, adaptation, distribution and reproduction c. in any medium or format, as long as you give appropriate credit to the original author(s) and the source, provide a link to the Creative Commons license, and indicate if changes were made. The images or other third party material in this article are included in the article's Creative Commons license, unless indicated otherwise in a credit line to the material. If material is not included in the article's Creative Commons license and your intended use is not permitted by statutory regulation or exceeds the permitted use, you will need to obtain permission directly from the copyright holder. To view a copy of this license, visit http://creativecommons.org/licenses/by/4.0/. 
substrates' specificity and therefore, functionality. First, mTORC1 binds specifically to Raptor and controls mostly protein synthesis and autophagy. Second, mTORC2 specifically binds to Rictor and phosphorylates the Serine 473 residue (Ser473) in Akt kinase to mediate neuronal survival. Among other functions, mTORC2 controls actin polymerization and, as a consequence, it is required for LTP and LTD induction to mediate synaptic strength $^{16,17}$.

Synaptic plasticity is impaired in HD patients ${ }^{18}$ and mouse models ${ }^{19-21}$. These plastic alterations correlate well with mTOR/Akt signaling axis impairment. For example, several HD mouse models show increased phosphorylation levels of striatal mTOR and the mTORC2 substrate Akt at the Ser473 residue ${ }^{22-24}$. Interestingly, PHLPP1 (PH domain leucine-rich repeat protein phosphatase 1), the phosphatase that dephosphorylates the Ser473 residue in $\mathrm{Akt}^{25,26}$, is decreased in the putamen of HD patients and in the striatum of HD mice ${ }^{23}$. Moreover, mTORC2-regulator protein Rictor, but not mTORC1-regulator protein Raptor, is increased in the striatum of HD mouse models and in the putamen of HD patients ${ }^{24}$. This evidence suggested the activation of a compensatory mechanism to counteract mhtt toxicity promoting neuronal survival. However, the exacerbation on the phosphorylation status of mTOR pathway components could eventually be counterproductive for synaptic function ${ }^{27,28}$.

In our previous work, we described RTP801/REDD1, an mTOR/Akt modulator, as a mediator of mhtt toxicity in in vitro models of HD. We also showed that RTP801 was elevated in the putamen and iPSC from HD patients ${ }^{29}$. RTP801, by interacting with TSC1/2 complex, regulates Rheb promoting its GDP-bound form. Rheb-GDP is not able to promote mTOR kinase activity of both mTORC1 and 2 complexes, inactivating S6 kinase and Akt activities, as readouts of mTORC1 and mTORC2, respectively. This mechanism was described to mediate neuronal death in Parkinson's disease (PD) models ${ }^{30,31}$. However, mTOR is hyperphosphorylated in the striatum of both HD mouse models and patients and therefore, the mechanism by which RTP801 contributes to the specific mTOR/Akt axis overactivation and how this is translated to impaired plasticity has not been elucidated yet. For this reason, here we investigated whether mhtt-induced RTP801 increase could alter mTOR/Akt signaling at a synaptic level and mediate motor impairment in HD.

\section{Results}

\section{Ectopic mhtt increases RTP801 at soma and dendritic} spines in rat cortical primary neurons

We have previously shown that overexpression of the pathogenic exon-1 of mhtt elevates RTP801 protein levels in rat cortical neuronal cultures ${ }^{29}$. Here, we extended these studies by analyzing whether RTP801 increase also occurs in the dendrites. Given that striatal mono-cultures generally produce morphologically immature MSNs with low densities of dendritic spines ${ }^{32,33}$, the use of cortical primary cultures facilitated this analysis.

Hence, rat cortical primary neurons (DIV13) were transfected with eGFP, Q25, Q72-, or Q103-htt-expressing plasmids. Low efficiency of Lipofectamine-based transfection of neuronal cultures assured sparse eGFP labeling and a proper visualization of the transfected neurons and their morphology. Twenty-four hours after transfection, neurons were stained against eGFP and endogenous RTP801 and dendritic F-actin puncta were visualized with phalloidin (Fig. 1a). Transfected neurons did not show signs of death, as judged by the Hoechst nuclear staining (Fig. 1a, merge panels). RTP801 showed somatic and dendritic expression and was also found within the dendritic spines, as indicated by the yellow arrowheads. The overexpression of exon-1mhtt (Q72 and Q103 pathogenic forms) induced a reduction in spine density (Fig. 1b) along with an increase in somatic RTP801 (Fig. 1c), consistent with our previous work $^{29}$. Importantly, mhtt overexpression also increased around 50\% the levels of RTP801 protein at the remaining dendritic spines (Fig. 1d).

\section{RTP801 is increased in the synaptic compartment in the putamen of HD patients}

Our previous work showed that RTP801 was elevated in whole putamen lysates from HD patients ${ }^{29}$. To further investigate whether the increase in RTP801 also extends to synaptic contacts, we isolated crude synaptosomes from the putamen of non-affected (CT) and HD individuals (Table S1). We found that RTP801 was enriched at the synaptosome subcellular compartment, along with synaptic markers SV2A and PSD-95. However, phosphoSer473-Akt (P-Ser473-Akt) and Phospho-Ser235/236-S6 (P-Ser235/236-S6) were not significantly enriched in the crude synaptosomes (Supplementary Fig. S1). In agreement with our previous results, we detected higher levels of RTP801 in homogenates derived from HD putamen (Supplementary Fig. S1A). Still, in homogenates, we confirmed that mTOR activities were elevated in HD as judged by increased levels of P-Ser473-Akt and P-Ser235/ 236-S6 (Supplementary Fig. S1D, E). Interestingly, we observed that RTP801 was significantly increased in HD synaptosomes (Fig. 2b) in comparison to controls. As expected and in line with previous data ${ }^{34}$, postsynaptic marker PSD-95 was diminished in synaptosomes from HD patients and no differences were observed in presynaptic marker SV2A (Fig. 2b). No significant differences were found in P-Ser473-Akt or P-Ser235/236-S6 at the synaptosomal compartment between non-affected and HD individuals (Fig. 2b).

In order to correct protein levels for the remaining synapses, synaptosomal levels of each protein were 


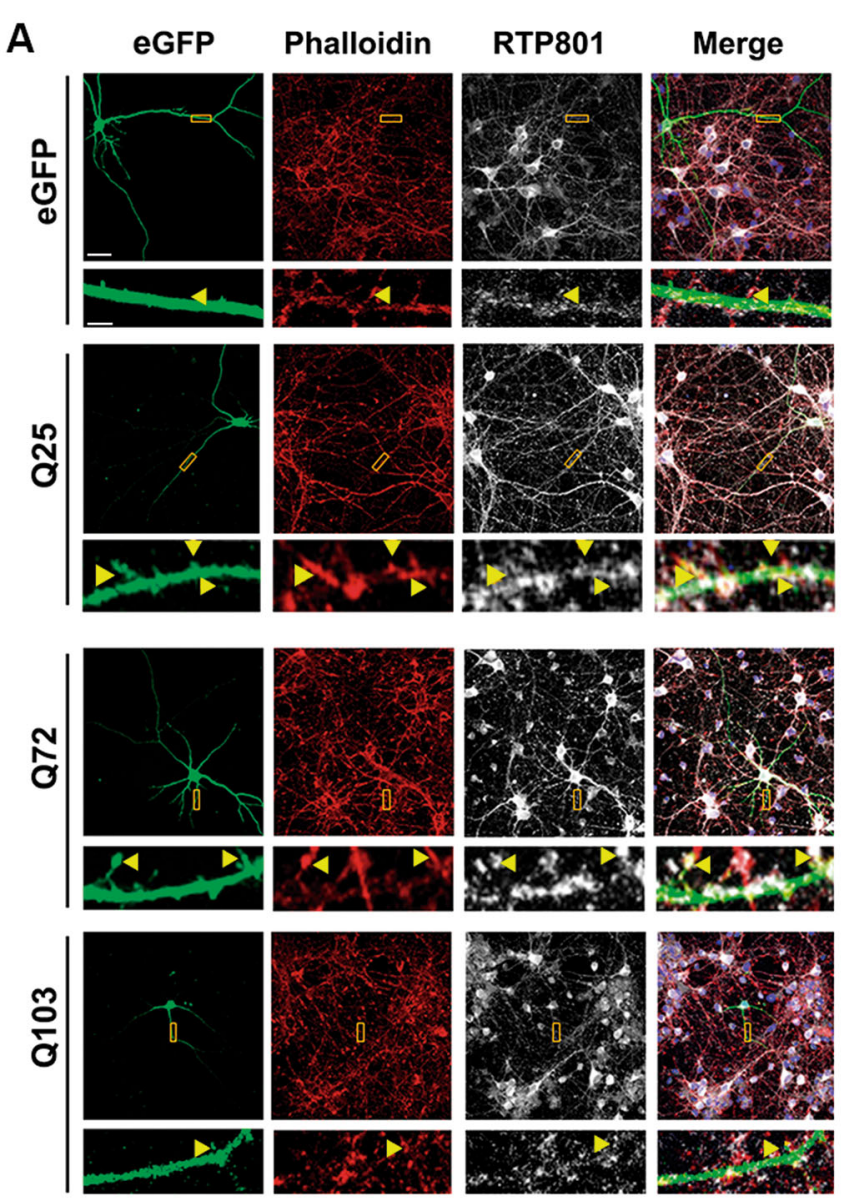

B
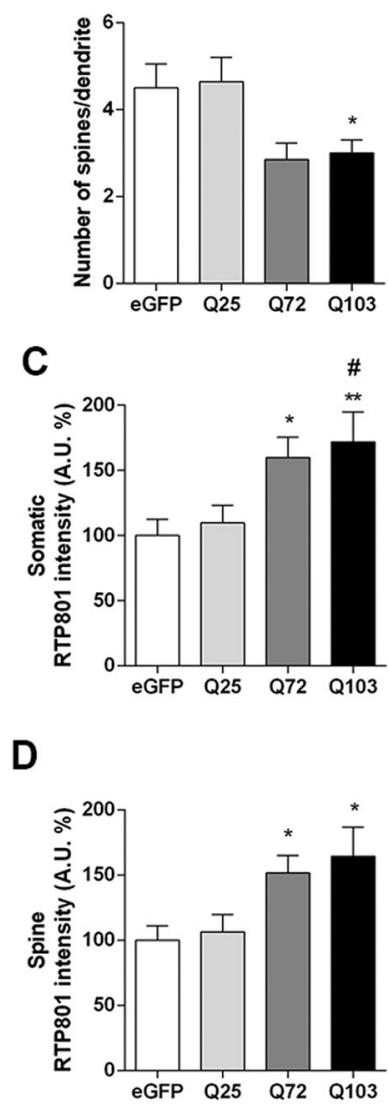

Fig. 1 Overexpression of exon-1 mhtt increases RTP801 protein levels in dendrites and in synaptic spines. a Rat cortical cultures at DIV13 were transfected with eGFP, Q25, Q72, or Q103 plasmids. Twenty-four hours after transfection cultures were fixed and stained against GFP (in green), RTP801 (in gray) and phalloidin (in red) to visualize the actin cytoskeleton. Nuclei were stained with Hoechst 33342 (in blue). Images were acquired by confocal microscopy. Yellow rectangles show digital zoom of dendrites with spines and yellow arrows show RTP801 staining at the puncta. Scale bar, $10 \mu \mathrm{m}$ and in higher magnification images, $1.5 \mu \mathrm{m}$. b Graphs display the number of spines scored for each $15 \mu \mathrm{m}$ dendrite-length and RTP801 staining intensity quantification at $\mathbf{c}$ the neuronal soma and at $\mathbf{d}$ the spines. Data are shown as percentage of RTP801 intensity (mean \pm SEM) of four independent experiments and were analyzed with one-way ANOVA followed by Dunnett's multiple comparisons test (for $\mathbf{b}$ and $\mathbf{c}$ ) or with Kruskal-Wallis with Dunn's multiple comparisons test (for $\mathbf{d}$ ) ${ }^{*} P<0.05$ and ${ }^{*} * P<0.01$ vs. eGFP and ${ }^{*} P<0.05$ vs. Q25, $n=13$ dendrites for eGFP, $n=10$ dendrites for Q25, Q72, and Q103 per experiment).

relativized to the levels of pre-synaptic marker SV2A (Fig. 2c) and postsynaptic marker PSD-95 (Fig. 2d). Hence, we confirmed that versus the SV2A- and PSD-95-synaptic proteins amount, RTP801 was increased in the HD group in comparison to controls. Furthermore, both P-Ser473Akt and P-Ser235/236-S6 were elevated in the PSD-95synaptic fraction of HD patients respect to unaffected individuals and showed the same tendency in the SV2Asynaptic fraction. These data are in line with the previously described impairment of the mTOR pathway in $\mathrm{HD}^{35-37}$.

Taken together, these results show that RTP801 is increased in the remaining synaptic terminals of HD patients' putamen, along with the mTOR substrates PSer473-Akt and P-Ser235/236-S6.
To determine whether these differences were regionspecific, we performed the same analyses in the prefrontral cortex, a less affected brain region in the disease. Similarly as in the putamen, RTP801, SV2A, PSD-95, P-Ser473-Akt, and P-Ser235/236-S6 were highly enriched in the synaptosomal compartment (Supplementary Fig. S2, Enrichment and Supplementary Fig. S3), although no differences in RTP801 levels were observed neither in homogenates (Supplementary Fig. S2, Homogenates) nor in synaptosomes (Supplementary Fig. S3) between HD and non-affected individuals. Only synaptosomal PSer235/236-S6 normalized to SV2A or to PSD-95 showed a significant decrease in HD samples in contrast to control cases (Supplementary Fig. S3C, D). 


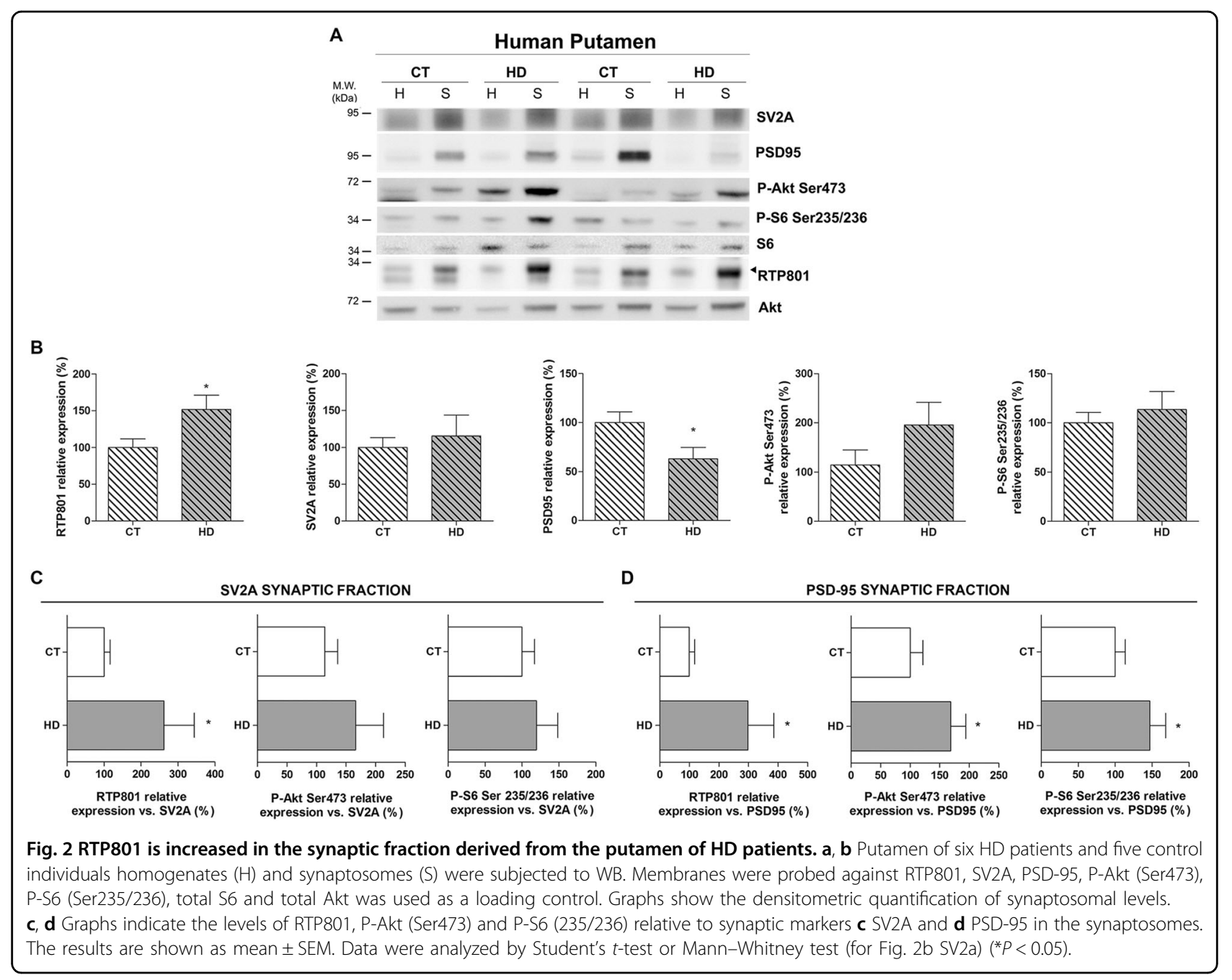

\section{RTP801 is increased in striatal synapses of HD mouse models}

To assess whether synaptic elevation of RTP801 could be reproduced in HD mouse models, we investigated the synaptic levels of RTP801 in the striatum of two different HD models. HD mice exhibit cognitive deficits before the onset of motor symptoms. To further study the synaptic machinery we isolated synaptosomes from the striatum of $\mathrm{Hdh}^{\mathrm{Q} 7 / \mathrm{Q} 11}$ and $\mathrm{R} 6 / 1$ mice at an age that already display motor learning and motor performance disturbances ${ }^{38-41}$. Given that these two mouse lines show a different disease speed progression, we isolated synaptosomes from 10month-old $\mathrm{Hdh}^{\mathrm{Q} 7 / \mathrm{Q} 111}$ (KI) (Fig. 3 and Supplementary Fig. S4) and 16-weeks old R6/1 (Fig. 4 and Supplementary Fig. S5).

Synaptosomal fractions of wild-type (WT) and HD mouse striata were enriched for RTP801, SV2A, PSD-95, and P-Ser235/236-S6 (Supplementary Figs. S4A-C, E and $\mathrm{S} 5 \mathrm{~A}-\mathrm{C}, \mathrm{E})$, as in human putamen. However, RTP801 protein levels did not differ between genotypes in either homogenates (Supplementary Figs. S4A and S5A) or synaptosomes (Figs. $3 \mathrm{~b}$ and 4b). PSD-95 displayed decreased protein levels in comparison to the WT group in $\mathrm{KI}$ and R6/1 mouse striatal synaptosomes (Figs. 3b and 4b). After correcting the synaptic loss by expressing protein levels relative to the synaptic markers SV2A (Fig. 3c and 4c) and PSD-95 (Fig. 3d and 4d), RTP801 was increased in the synaptic fraction of $\mathrm{KI}$ and R6/1 mice. Interestingly, only P-Ser473-Akt was also significantly increased at the synaptic fraction of KI mice, whereas in R6/1 mice, P-Ser235/236-S6 levels were also increased.

\section{RTP801 knockdown prevents motor-learning deficits in symptomatic $\mathrm{R} 6 / 1$ mice}

Given that we found alterations in the striatal synaptic protein levels of RTP801 in HD mouse models and human HD brains, we investigated whether RTP801 could contribute to plasticity dysfunction in HD. To answer this question, 9-week-old WT and R6/1 mice were bilaterally injected with AAV-shCtr or AAV-shRTP801 into the 


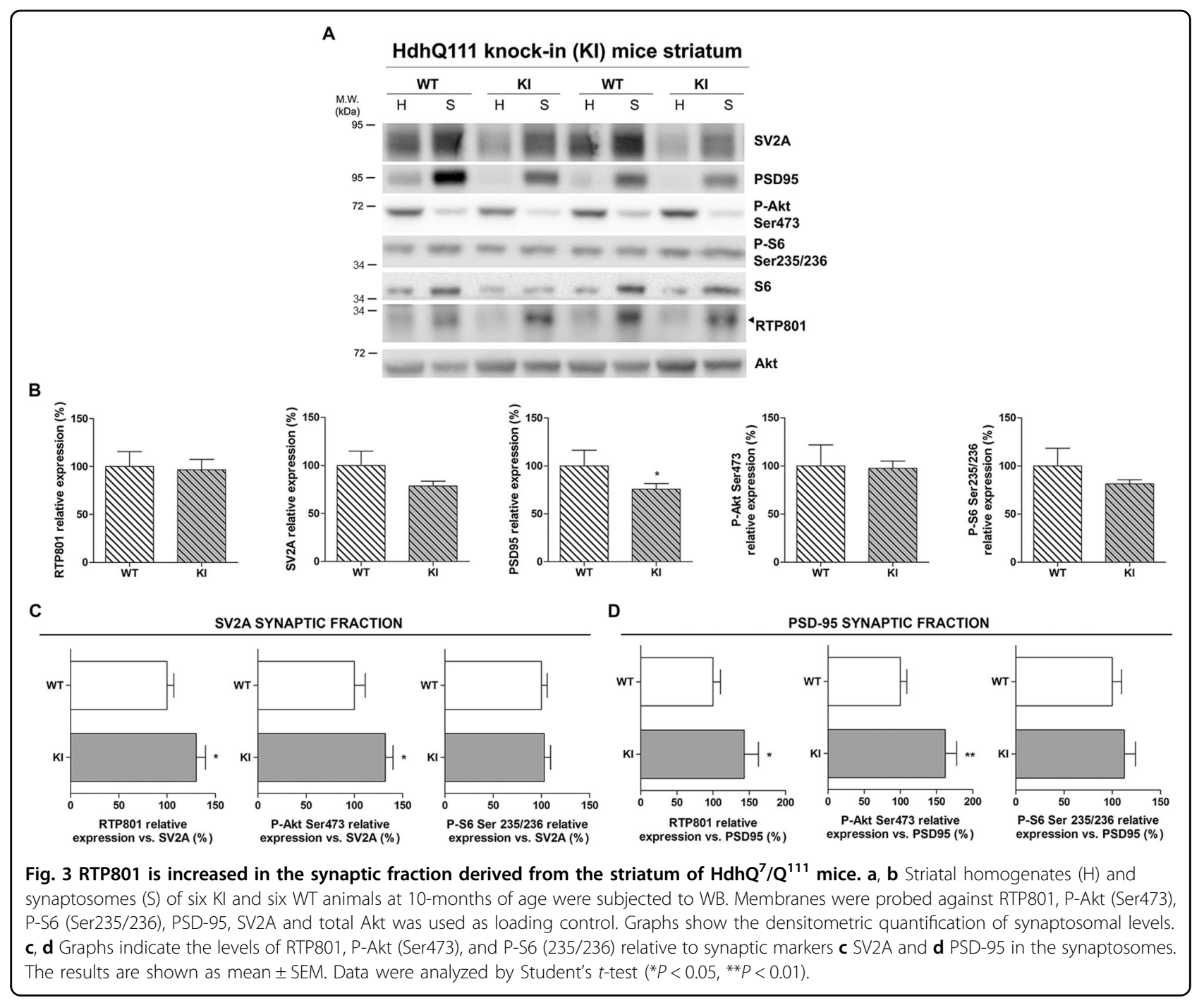

striatum and 5 weeks later motor learning, a corticostriatal-dependent function, was assessed using the accelerating rotarod.

Comparable GFP signal was detected in the striatum of WT and R6/1 mice confirming the transduction of AAVshCtr and AAV-shRTP801 (Fig. 5a). Moreover, striatal injections of AAV-shRTP801 reduced RTP801 protein levels between 25 and 30\%, both in WT and R6/1 mice (Fig. $5 b)$. The accelerating rotarod test showed that, as expected, shCtr-injected R6/1 animals performed poorly in this motor task in comparison to WT-shCtr-injected animals. Remarkably, RTP801 knockdown in the striatum prevented motor-learning deficits in the R6/1 mice as shown by the increased latency to fall, which was comparable to that in WT animals. Indeed, AAV-shCtr or AAV-shRTP801injected WT mice showed no differences in motorlearning skills (Fig. 5c). These results indicate that mhttinduced RTP801 synaptic increase contributes to motorlearning dysfunction in the R6/1 mouse model of HD.
RTP801 knockdown normalizes phospho-Akt Ser473 and enhances the expression of synaptic proteins in the R6/1 mouse striatum

To understand the pathological function of synaptic RTP801 and how its knockdown prevents motor-learning dysfunction, we analyzed downstream effectors of the mTOR pathway in striatal synaptic fractions obtained from WT and R6/1 mice transduced with AAV-shCtr or AAV-shRTP801 by WB (Fig. 6 and Supplementary Fig. S6, Enrichment).

Knocking down RTP801 affected neither the levels of P-Ser2448-mTOR nor the levels of P-Ser235/236-S6, as mTORC1 readout (Fig. 6a, b) but interestingly, it restored P-Ser473-Akt levels to basal levels both in homogenates and synaptosomes, with no significant effect in WT animals (Fig. 6c). Therefore, this result indicates that RTP801 regulates the phosphorylation levels of Serine 473 Akt in this pathological context.

According to previous studies, increased levels of Rictor and reduced levels of PHLPP1 phosphatase are 


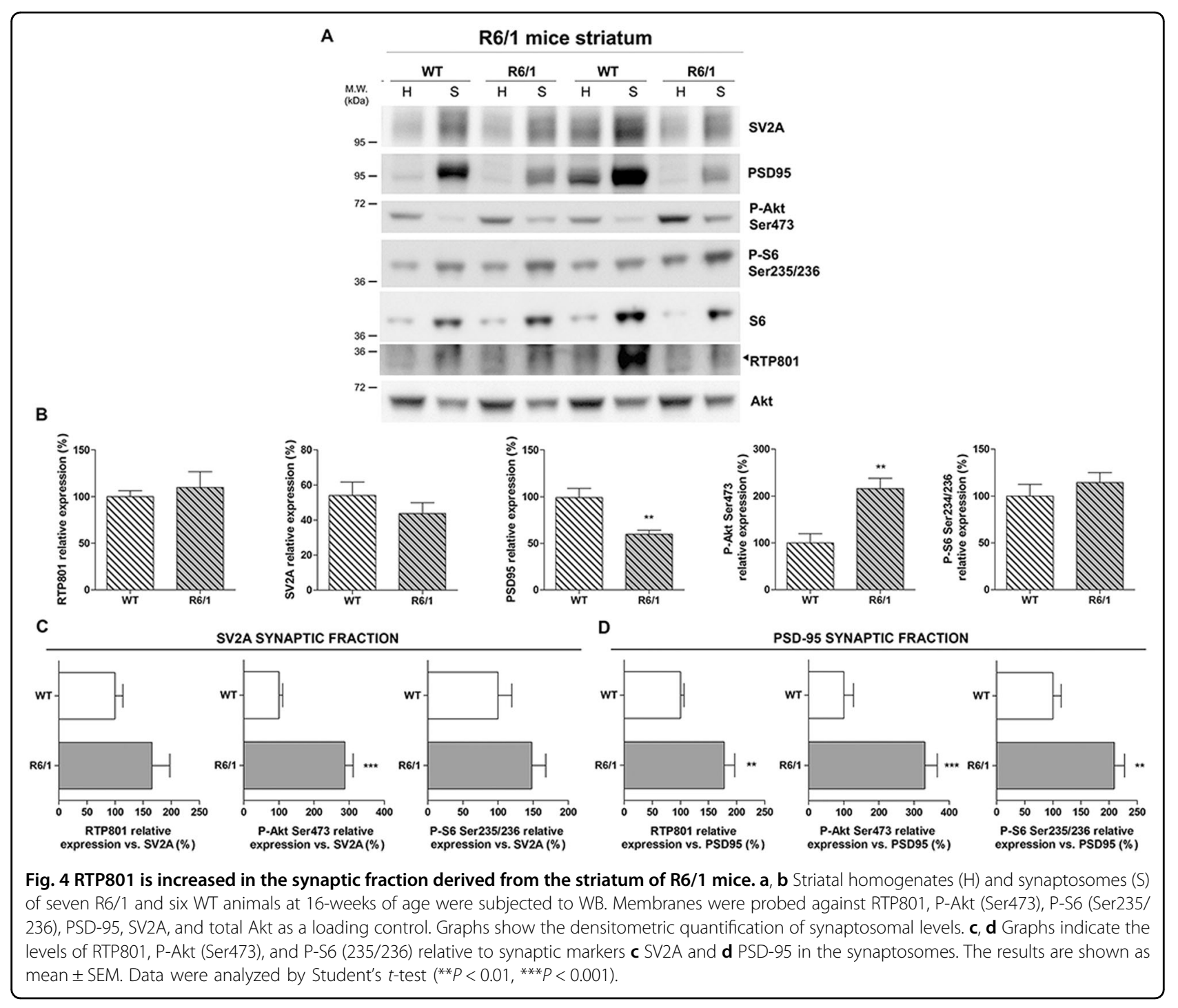

responsible for Akt hyperactivation in the R6/1 mouse model $^{23,24}$. Therefore, we investigated whether RTP801 knockdown in R6/1 mice could affect these two Akt modulators. Strikingly, RTP801 knockdown normalized specifically the synaptic levels of Rictor in R6/1 mice (Fig. 6d) without altering the levels of PHLPP1 (Fig. 6e). Hence, these results suggest that RTP801 knockdown prevents Akt hyperactivation by decreasing Rictor protein levels and thus downregulating the activity of mTORC2.

We next asked whether RTP801 downregulation could be translated to synaptic transmission modulation. Striatal RTP801 silencing induced an increase of AMPA receptor subunit GluA1 at the homogenate fraction in shRTP801injected R6/1 mice in comparison to shCtr-injected R6/1 mice, whereas non-significant tendencies were observed at the synaptic compartment (Fig. 7a).

One of the mechanisms contributing to synaptic impairment in the striatum of HD mouse models is the imbalance between BDNF receptors, TrkB and $\mathrm{p} 75^{\mathrm{NTR}} 42,43$. We found that RTP801 silencing increased TrkB levels in both homogenates and synaptosomes of R6/1 mice striatum in comparison to their shCtr-injected R6/1 littermates (Fig. 7b), whereas p $75^{\text {NTR }}$ was not modified in any compartment analyzed (Fig. 7c). Neither synaptosomal levels of PSD-95 (Fig. 7d) nor NR1 (Fig. 7e) were affected by knockdown of RTP801.

This evidence indicates that striatal RTP801 knockdown prevents R6/1 motor-learning deficit by decreasing Rictor levels, normalizing the bulk of P-Ser473-Akt and possibly, by enhancing postsynaptic signaling.

Altogether, the results indicate that RTP801 is increased in the synapses from the striatum, the most susceptible brain area to mhtt toxicity in both humans and HD mouse models, and its upregulation contributes to impair motorlearning-related synaptic plasticity (Fig. 8). 


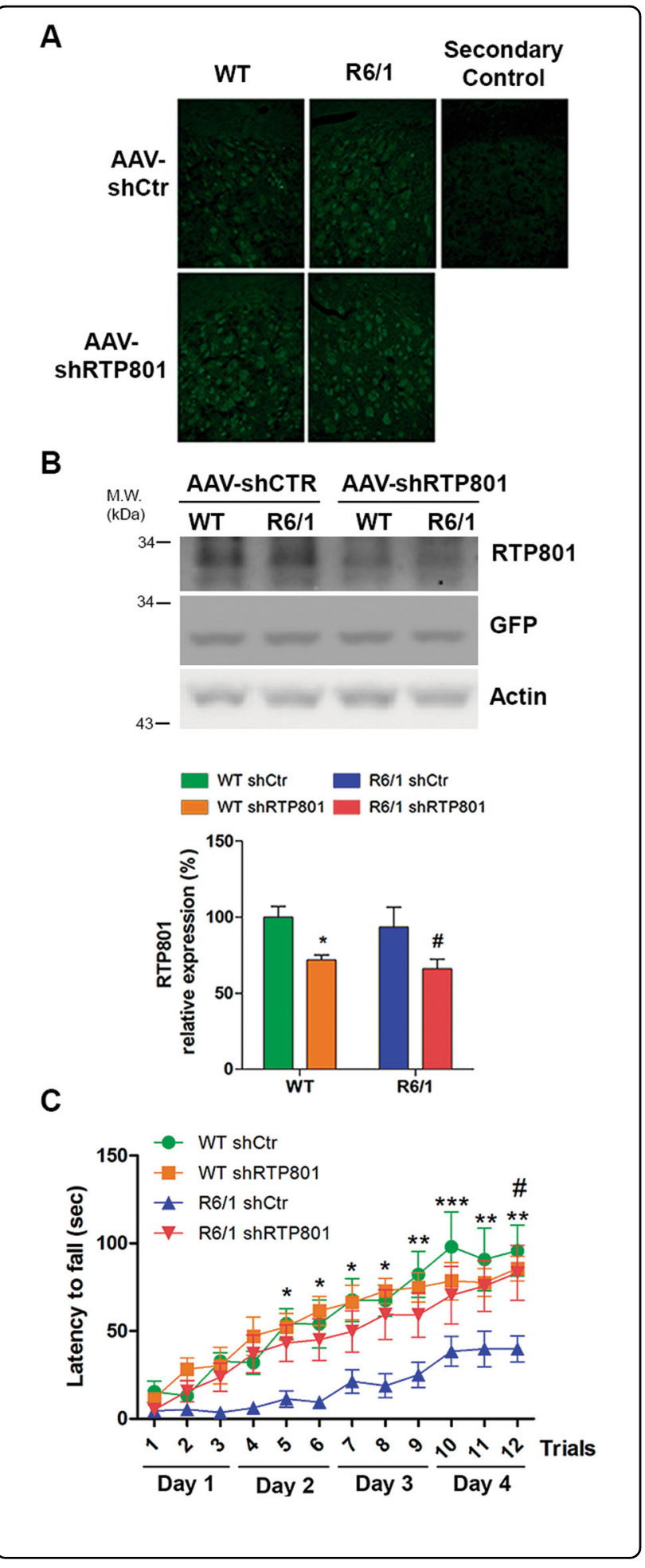

\section{Discussion}

HD is characterized by aberrant synaptic plasticity and progressive and selective loss of neuronal subpopulations $^{10,11,19,44,45}$, in which mTOR plays a critical role ${ }^{13}$. In this work, we have investigated whether mhtt-induced
Fig. 5 Striatal RTP801 knockdown preserves motor learning in symptomatic R6/1 mouse. a GFP immunofluorescence confirmed the transduction of AAV-shCtr and AAV-shRTP801 in 9-week-old WT and R6/1 mice striatal cells. A secondary control was performed without the incubation of primary antibody (anti-GFP). Scale bar $450 \mu \mathrm{m}$. b Striatal lysates of WT and R6/1 injected with AAV-shCtr ( $n=6 \mathrm{WT}$ and $n=6 \mathrm{R} 6 / 1)$ or AAV-shRTP801 ( $n=6 \mathrm{WT}$ and $n=7 \mathrm{R} 6$ / 1) were subjected to WB. Membranes were probed against RTP801, GFP, and actin as a loading control. Graph show the densitometric quantification of RTP801 signal. Data are shown as a mean \pm SEM and were analyzed with two-way ANOVA followed by Bonferroni's multiple comparisons test for post hoc analyses ( ${ }^{*} P<0.05$ vs. WT AAV-shCtr $\# P<0.05$ vs. R6/1 AAV-shCtr). c Five weeks after adenoassociated transduction, motor learning was assessed in the same animals by the accelerating rotarod ( $n=8$ WT-shCtr, $n=8$ WT shRTP801, $n=9$ R6/1-sh(tr, $n=9$ R6/1-shRTP801). The graph shows the latency to fall as the mean of three trials tested each day. Values are expressed as mean \pm SEM and were analyzed with two-way ANOVA followed by Bonferroni's multiple comparisons test for post hoc analyses $\left({ }^{*} P<0.05,{ }^{*} P<0.01\right.$, and ${ }^{* *} P<0.001$ R6/1-shCtr vs. WT-shCtr and ${ }^{\#} P<0.05$ R6/1-shRTP801 vs. R6/1-shCtr).

RTP801 increase contributes to the impairment of synaptic plasticity in HD. Our results show that RTP801 is elevated in cultured neurons' synapses overexpressing mhtt exon-1. Moreover, putamen, but not cortex, of HD patients displays increased levels of synaptic RTP801 respect to unaffected individuals. In addition, in HD mouse models, RTP801 is highly enriched in the striatal synaptic compartment when compared to WT littermates. Notably, RTP801 knockdown in the striatum of R6/1 mice prevents motor-learning deficits, which is accompanied by a synaptic restoration of Rictor and PSer473-Akt levels and an increase of GluA1 and TrkB receptors in the striatum.

We first demonstrated that mhtt elevates RTP801 protein in the soma and in the dendritic spines of primary cortical neurons, in accordance with our previous results obtained in differentiated $\mathrm{PC} 12$ cells $^{29}$. Interestingly, mhtt-induced RTP801 upregulation courses along with a reduction in spine density. These results are in line with extensive work reporting the loss of dendritic spines in neurons overexpressing $\mathrm{mhtt}^{46,47}$ and suggest that RTP801 synaptic increase contributes to this phenomena.

In human HD putamen, RTP801 protein levels were not only increased in whole lysates, as we previously described $^{29}$, but also in synaptosomes. Structural and morphological synaptic alterations in striatal medium-sized spiny neurons ${ }^{48,49}$ and loss of dendritic spines in HD postmortem brains have been widely reported ${ }^{34,48,49}$. In agreement with our findings, PSD-95 is also decreased in the striatum of HD patients ${ }^{34}$. Given that we observed that RTP801 is enriched in the synaptic fraction and is highly accumulated in HD synaptic contacts in the striatum, we speculated that synaptic RTP801 in the 


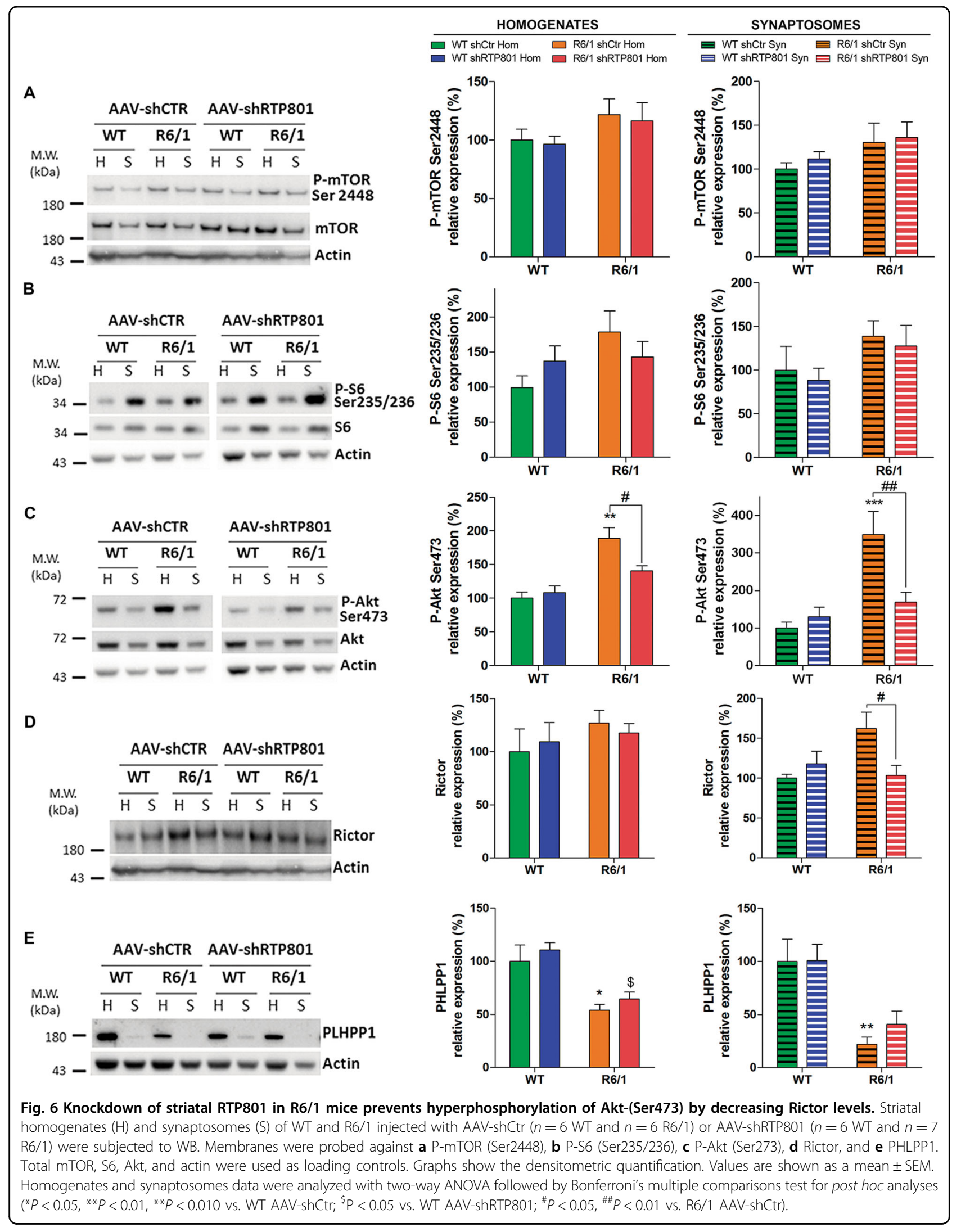




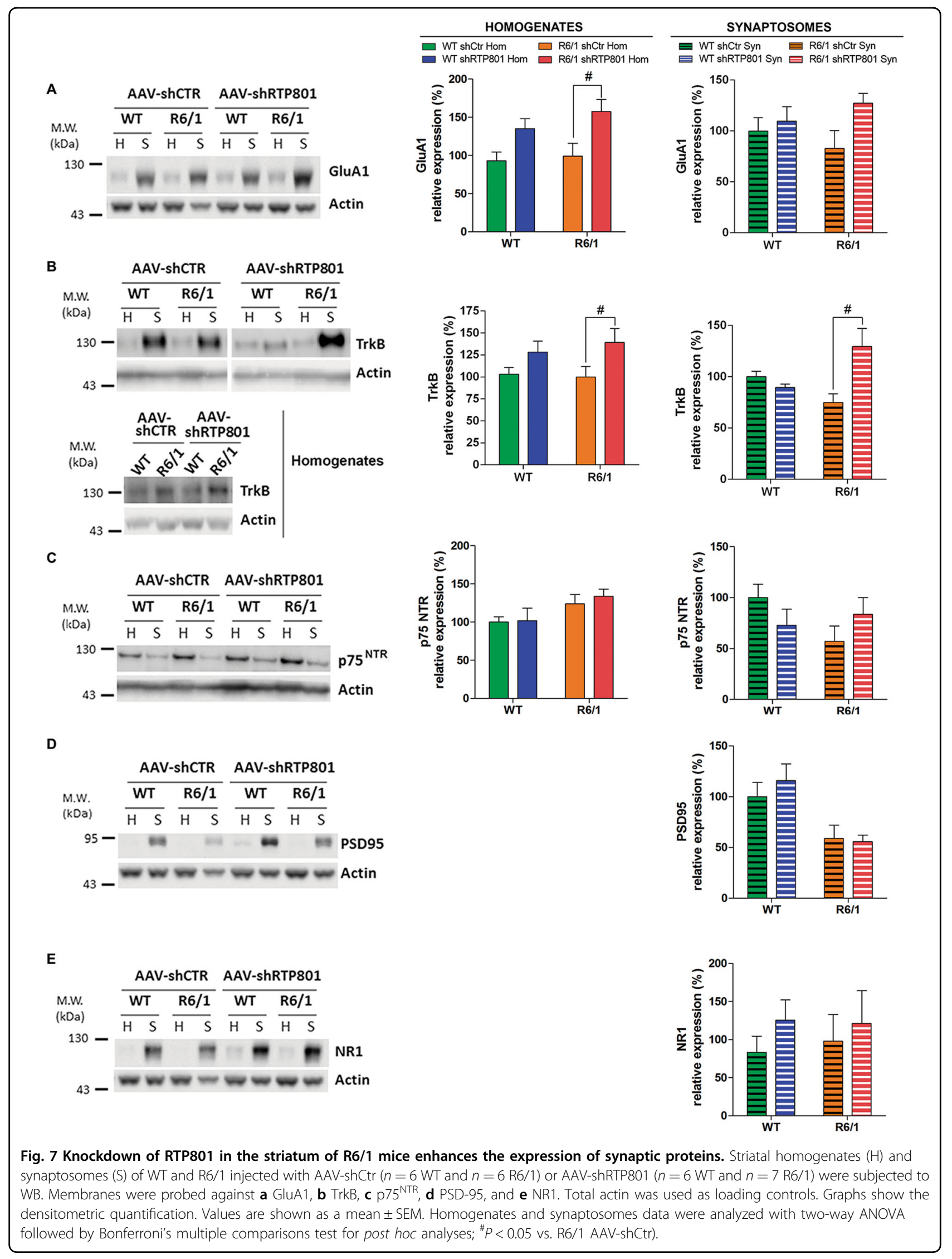




\section{WT mice}

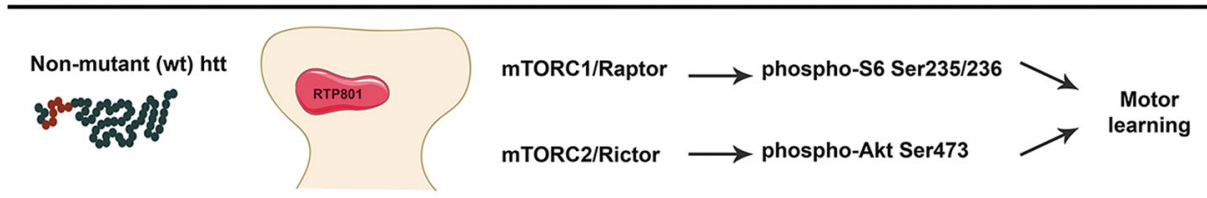

R6/1 mice

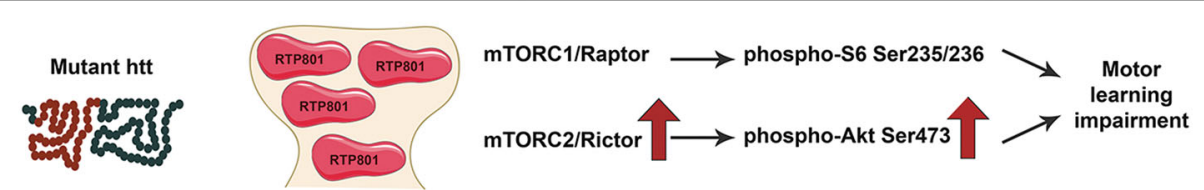

\section{R6/1 mice + RTP801 knockdown}

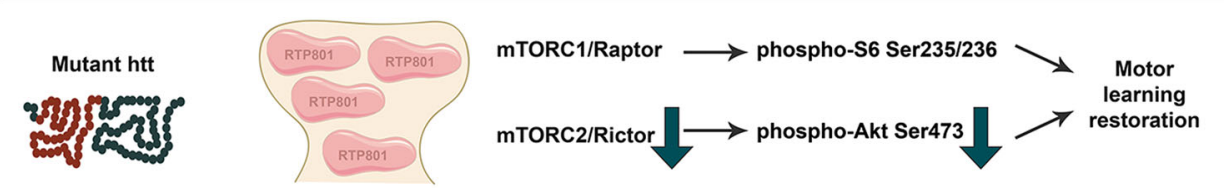

Fig. 8 RTP801 knockdown prevents corticostriatal learning deficits. In comparison to WT mice, R6/1 mice display increased RTP801 at the striatal synapse along with increased Rictor and P-Akt (Ser473) and motor-learning deficits. RTP801 silencing preserves corticostriatal motor-learning function by decreasing the levels of Rictor and P-Akt Ser473 and enhancing postsynaptic signaling by increasing GluA1 and TrkB receptors.

remaining striatal dendritic spines could have a role in the altered plasticity associated with this neurodegenerative process. In addition, we found that RTP801 is also enriched in human cortical synapses, but no differences were found between controls and HD patients in the synaptic fraction. Even though morphological alterations in HD prefrontal cortical pyramidal neurons have been described $^{18}$, our results indicate that RTP801 elevation is specific for the striatum. In line with this, neither the postsynaptic marker PSD-95 nor the pre-synaptic marker SV2A were altered in the prefrontal cortex. Our results thus suggest that may be intrinsic neuronal properties modulate the effect of mhtt depending on the subcellular type.

In HD pathogenesis, synaptic impairment precedes motor symptoms ${ }^{7-9}$. In fact, HD mouse models are reported to exhibit cognitive deficits before the appearance of motor symptoms, indicating that abnormal synaptic plasticity occurs at early stages of the disease also in mouse models ${ }^{50,51}$.

In accordance with the lack of neuronal death in HD mouse models ${ }^{38,39,52-54}$, striatal levels of RTP801 in homogenates were not altered either in R6/1 or KI mice. However, specifically analyzing protein levels in synaptosomal preparations, we concluded that mhtt leads to a localized aberrant accumulation of RTP801 in synapses that could contribute to plasticity impairment in the pathology. In fact, structural alterations at the morphology and/or spine number in the striatum of the $\mathrm{R} 6 / 1^{55}$ and the $\mathrm{KI}^{56}$ have been reported. This observation is also extended to other HD murine models such as $\mathrm{N}$-terminal fragment R6/2 mice ${ }^{57,58}$ and transgenic full-length htt YAC128 mice ${ }^{59}$. Supporting our results, levels of PSD-95, as a synaptic marker, is reduced in those HD animal models $^{51,60,61}$.

To evaluate the corticostriatal function, responsible for motor learning ${ }^{62}$, R6/1 mice transduced with AAV-shCtr or AAV-shRTP801 were subjected to the accelerating rotarod test. Interestingly, silencing RTP801 in the striatum of R6/1 mice preserves their performance in the accelerating rotarod, comparable to WT mice, indicating that RTP801 knockdown retains the ability to learn new motor skills in the HD mice. The fact that silencing RTP801 in WT animals does not affect their performance at this paradigm may suggest that remaining basal levels of RTP801 are sufficient to maintain proper motorlearning plasticity and, therefore, corticostriatal functionality. Highlighting the function of RTP801 in synaptic plasticity, its downregulation in the Substantia Nigra pars compacta has also been shown to restore motor-learning skills in a PD mice model subjected to chronic stress ${ }^{63}$.

The only known function of RTP801 is as a mTOR signaling repressor. In PD studies using cellular and animal models, RTP801 upregulation upon cell stress represses mTOR and subsequently inhibits Akt prosurvival signals, triggering neuronal death. In PD cellular models and nigral neurons of PD brains, RTP801 increases along with a decrease in the phosphorylation of Akt and $\mathrm{S6}^{30,31}$. Indeed, in HD, alterations in the activity of both mTORC1 ${ }^{37}$ and $\mathrm{mTORC} 2^{24}$ have been described. 
Here, we confirm that both mTOR complexes display aberrant activity and, importantly, that alterations found at total cell level are extended to the synaptic compartment.

Unlike in PD models, we show that mhtt increases RTP801 despite the hyperphosphorylation of Akt at Ser473 residue in the synaptosomes. Interestingly, PSer235/236-S6 displays the same trend. The fact that RTP801 levels are increased in HD models may also be a consequence of mTOR pathway hyperactivity, since RTP801 protein synthesis is mTORC1-dependent ${ }^{64}$.

Supporting its synaptic role, RTP801 silencing also alters the levels of mTOR pathway-associated protein components and enhances the expression of postsynaptic proteins. Our data indicate that RTP801 downregulation in R6/1 mice striatum prevents Akt hyperphosphorylation at Ser473 residue, both at homogenates and synaptosomes. Overactivation of Akt has been proposed to be a pro-survival response to counteract mhtt toxicity in the disease $^{22-24}$ However, some evidences have also pointed out that synaptic plasticity alterations occur along with hyperphosphorylation of $\mathrm{Akt}^{65}$ and that sustained overactivation of Akt could be detrimental for cell survival and synaptic function ${ }^{66,67}$. Moreover, the function of mTOR complexes can differ between neuronal compartments. For instance, mTORC2 at the soma mediates the activation of Akt to ensure neuronal survival, whereas mTORC2 close to the synapse mediates the activation of PKC, responsible for the polymerization of actin and receptor trafficking (reviewed in ref. ${ }^{68}$ ). Thus, RTP801 function may be different between cell compartments. Altogether, this evidence confirms the regulation of RTP801 over mTORC2-dependent Akt phosphorylation but, in the presence of mhtt, the regulation is not negative.

Phosphorylation levels of Akt at Ser473 depend on a proper activity of the mTOR kinase complexed with Rictor $^{69}$ and the phosphatase PHLPP1 ${ }^{25,26}$, that specifically dephosphorylates this residue. In fact, in HD, increased Rictor and decreased PHLPP1 levels are both responsible for Akt overactivation ${ }^{23,24}$. In line with that, we found that synaptic up regulation of Rictor in the R6/1 mice is sensitive to RTP801 levels since RTP801 knockdown specifically reduces synaptosomal Rictor levels without affecting PHLPP1. Still the regulation of RTP801 towards Rictor or whether it exists any feedback mechanisms over Akt phosphorylation remains uncertain.

Interestingly, we observed that RTP801 knockdown in the striatum elevated the levels of AMPA subunit receptor GluA1. AMPA receptors normally are heterotetramers composed mostly by calcium impermeable GluA2 subunits. However, GluA1 subunit confers calcium permeability to the pore, allowing signaling activation and synaptic regulation ${ }^{70-72}$. Therefore, we speculate that this GluA1 up regulation could affect the composition of the AMPA receptors and promote synaptic strength. Accordingly, RTP801 knockdown elevated TrkB levels in R6/1 homogenates and the same trend was observed in the synaptosomal fraction, with no changes in $\mathrm{p} 75^{\mathrm{NTR}}$. Both TrkB and $\mathrm{p} 75^{\mathrm{NTR}}$ are BDNF receptors, although each receptor activates different signaling pathways. BDNF mediates neuronal survival by the activation of $\operatorname{TrkB}$ receptor ${ }^{73}$ whereas $\mathrm{p} 75^{\mathrm{NTR}}$ can activate apoptotic signals, leading to neuronal death ${ }^{42,74-}$ 77. Since TrkB receptor is coupled to PI3K/Akt cascade, RTP801 could modulate Akt activation and contribute to synaptic dysregulation by modulating TrkB receptor levels. Indeed, imbalance of TrkB and $\mathrm{p} 75^{\mathrm{NTR}}$ has been proposed to mediate impaired plasticity in $\mathrm{HD}^{42,78}$. Hence, increased TrkB and GluA1 levels, as a result of RTP801 silencing, could promote neuroprotection and enhancement of synaptic transmission ${ }^{73,78}$.

\section{Conclusions}

Our results indicate that in vivo downregulation of striatal RTP801 prevents motor-learning deficits in R6/1 mouse model, in part by preventing Akt hyperphosphorylation at the Ser473 residue via Rictor downregulation and by increasing postsynaptic GluA1 and TrkB receptors. Hence, RTP801 emerges as a promising target for future therapeutic strategies to prevent or at least halt the progression of $\mathrm{HD}$.

\section{Methods}

\section{Cortical primary neurons}

Primary cortical cultures were obtained from rat Sprague-Dawley embryos at day 18 (E18) as previously described $^{29}$. Briefly, cortices were dissected out, dissociated in $0.05 \%$ trypsin (Sigma-Aldrich) and maintained in Neurobasal medium supplemented with B27 and $2 \mathrm{mM}$ GlutaMAX $^{\mathrm{TM}}$ (all from Thermo Fisher Scientific) in a 5\% $\mathrm{CO}_{2}$ humified atmosphere at $37^{\circ} \mathrm{C}$. For imaging experiments, neurons were plated at a density of 300 cells $/ \mathrm{mm}^{2}$ on poly-L-lysine-coated (Sigma-Aldrich) in seeding medium containing MEM medium (Thermo Fisher Scientific) supplemented with $10 \%$ heat-inactivated horse serum (Sigma-Aldrich). After $60 \mathrm{~min}$, medium was replaced for supplemented Neurobasal. Medium was replenished one third every 7 days.

Transfection of the constructs eGFP, Q25, Q72, and Q103 and immunocytochemistry of neuronal cultures were performed as described elsewhere ${ }^{79}$. Briefly, cultures were incubated with the primary antibody mouse monoclonal against GFP (1:1000, Santa Cruz Biotechnology, \#sc-101525) and rabbit polyclonal against RTP801 (1:150, Proteintech Group Inc., \#10638-1-AP). The secondary antibodies used were goat anti-Mouse IgG $(\mathrm{H}+\mathrm{L})$ conjugated to AlexaFluor ${ }^{\text {TM }} 488$ or goat anti-Rabbit IgG $(\mathrm{H}+\mathrm{L})$ conjugated with AlexaFluor ${ }^{\mathrm{TM}} 633$ (all from 
Thermo Fisher Scientific, \#A11017 and \#A21072, respectively). For nuclear staining, nuclei were revealed with Hoechst 3342 (Thermo Fisher Scientific) diluted 1:5000 along with the secondary antibody incubation. In the case of actin filamentous labeling, AlexaFluor ${ }^{\mathrm{TM}} 568$ Phalloidin (1:10000, Thermo Fisher Scientific, \#A12380) was incubated along with the secondary antibody and Hoechst 3342 (1:5000, Thermo Fisher Scientific, \#scH3570). Spine density was calculated by scoring the number of spines for each $15 \mu \mathrm{m}$ dendrite-length of at least ten dendrites per condition, in four independent experiments. ImageJ was used to quantify RTP801 intensity as the integrated density in the soma and in the dendrites.

\section{HD mouse models}

R6/1 transgenic mice expressing the exon- 1 of mhtt with 145 CAG repeats were obtained from Jackson Laboratory and maintained in B6CBA background. $\mathrm{Hdh}^{\mathrm{Q} 7}$ WT mice, with 7 CAG repeats, and $\mathrm{Hdh}^{\mathrm{Q} 111}$ knock-in mice, with targeted insertion of 109 CAG repeats that extends the glutamine segment in murine htt to 111 residues, were maintained on a $\mathrm{C} 57 \mathrm{Bl} / 6$ genetic background. Male and female $\mathrm{Hdh}^{\mathrm{Q} 7 / \mathrm{Q} 111}$ heterozygous were intercrossed to generate age-matched $\mathrm{Hdh}^{\mathrm{Q} 7 / \mathrm{Q}^{7}}$ WT and $\mathrm{Hdh}^{\mathrm{Q} 7 / \mathrm{Q} 111}$ knock-in littermates. Age of the mice was specified in each figure legend according to the experimental approach requirements. All mice used were males and were housed together in numerical birth order in groups of mixed genotypes. Data were recorded for analysis by microchip mouse number. Animals were housed with access to food and water ad libitum in a colony room kept at $19-22{ }^{\circ} \mathrm{C}$ and $40-60 \%$ humidity, under a $12: 12 \mathrm{~h}$ light/dark cycle. All procedures were carried out in accordance with the National Institutes of Health Guide for the Care and Use of Laboratory Animals and approved by the local animal care committee of the Universitat de Barcelona, following European (2010/63/UE) and Spanish (RD53/2013) regulations for the care and use of laboratory animals.

\section{Human postmortem tissue}

Postmortem striatal and frontal cortical brain tissues from control and HD patients were obtained from the Neurological Tissue Bank (Biobank-HC-IDIBAPS) thanks to Dr. Ellen Gelpi collaboration following the guidelines the approval of the local ethics committee at recruitment, an informed written consent was obtained. (see Supplementary Table 1 ).

\section{Crude synaptosomal preparations}

Synaptosomes were prepared from mouse striata and human putamen and frontal cortex as previously described elsewhere with minor modifications. Briefly, dissected tissue was homogenized in Krebs-Ringer (KR) buffer $(125 \mathrm{mM} \mathrm{NaCl}, 1.2 \mathrm{mM} \mathrm{KCl}, 22 \mathrm{mM} \mathrm{NaHCO}, 1 \mathrm{mM}$ $\mathrm{HaH}_{2} \mathrm{PO}_{4}, 1.2 \mathrm{mM} \mathrm{MgSO}, 1.2 \mathrm{mM} \mathrm{CaCl} 2,10 \mathrm{mM}$ glucose $(\mathrm{pH}=7.4))$ supplemented with $0.32 \mathrm{M}$ Sucrose. Then, samples were subjected to a first centrifugation at $1000 \times g$ for $10 \mathrm{~min}\left(4^{\circ} \mathrm{C}\right)$ to discard debris. A sample of the supernatant was kept as the homogenate fraction. After, the supernatant was subjected to a second centrifugation at $16,000 \times g$ for $15 \mathrm{~min}\left(4^{\circ} \mathrm{C}\right)$ to obtain the crude synaptosomal fraction. The pellet was finally resuspended in KR $0.32 \mathrm{M}$ sucrose buffer and subjected to WB to analyze protein content.

\section{Western blotting}

Homogenate and synaptosomal protein analysis was performed by western blotting as previously described ${ }^{80}$. The following primary antibodies were used: Akt rabbit polyclonal (1:1000, Cell Signaling, \#4691), Akt-phosphoSer473 rabbit polyclonal (1:1000, Cell Signaling, \#4060), GFP rabbit polyclonal (1:1000, Santa Cruz Biotechnology, \#sc-8334), GluA1 rabbit polyclonal (1:1000, Merck Millipore, \#ABN241), mTOR rabbit polyclonal (1:1000, Cell Signaling, \#2971), mTOR-phospho Ser2448 (1:1000, Cell Signaling, \#2972), anti-NR1 mouse monoclonal (1:1000, Chemicon, \#MAB363), p75 ${ }^{\text {NTR }}$ rabbit polyclonal (1:1000, Promega, \#G323A), PHLPP1 rabbit polyclonal (1:500, Cayman Chemical, \#10007191), PSD-95 mouse monoclonal (1:1000, Thermo Fisher Scientific, \#MA1-045), Rictor rabbit polyclonal (1:1000, Cell Signaling, \#2140), RTP801 (1:500, Proteintech Group Inc., \#10638-1-AP), RPS6 mouse monoclonal (1:500, Cell Signaling, \#2317), RPS6-phospho-Ser235/236 rabbit polyclonal (1:1000, Cell Signaling, \#4858), SV2A mouse monoclonal (1:1000, Santa Cruz Biotechnology, \#sc-376234) and TrkB mouse monoclonal (1:1000, BD Biosciences, \#610102). Then, membranes were incubated with the corresponding secondary antibodies: goat anti-Mouse $\operatorname{IgG}(\mathrm{H}+\mathrm{L})$ and goat anti-Rabbit IgG $(\mathrm{H}+\mathrm{L})$ conjugated to horseradish peroxidase (1:10,000, Thermo Fisher Scientific, \#31430 and \#31460, respectively). Loading control was obtained incubating with anti- $\beta$-actin-horseradish peroxidase conjugated (1:100,000, Sigma-Aldrich, \#A3854-200U). Chemiluminescent images were acquired using a LAS-3000 imager (Fuji) or ChemiDoc (Bio-Rad) imaging systems and quantified by computer-assisted densitometric analysis (ImageJ software).

\section{Intrastriatal injection of adeno-associated vectors}

To knockdown RTP801 expression, shRNA verified scrambled sequence (5'-GTGCGTTGCTAGTACCAAC$3^{\prime}$ ) and the one against RTP801 (5'-AAGACTCCTCA TACCTGGATG-3') were cloned into a rAAV2/8-GFP adenoviral vector at restriction sites BamHI at $5^{\prime}$ and Agel at the $3^{\prime}$. The rAAV2/8 plasmids and infectious AAV viral 
particles containing GFP expression cassette with shCtr or shRTP801 were generated by the Vectors Production Unit from the Center of Animal Biotechnology and Gene Therapy at the Universitat Autònoma de Barcelona. Nineweek-old WT and R6/1 mice were subjected to bilateral intrastriatal injections of rAVV2/8-expressing shRTP801 or control shRNA. Animals were deeply anesthetized with a mixture of oxygen and isoflurane $(4-5 \%$ for induction and $1-2 \%$ for maintaining anesthesia) and placed in a stereotaxic apparatus for bilateral intrastriatal injections $\left(2 \mu \mathrm{l} ; 15 \times 10^{9}\right.$ genomic copies). Two injections were performed in the striatum at the following coordinates relative to bregma: (1) anteroposterior (AP), +0.8 ; mediolateral (ML), $+/-1.8$; and profundity, $-2.6 \mathrm{~mm}$ and (2) $\mathrm{AP},+0.3 ; \mathrm{ML},+/-2$; and profundity, $-2.6 \mathrm{~mm}$, below the dural surface. Viral vectors were injected using a $10 \mu \mathrm{l}$-Hamilton microliter syringe at an infusion rate of $200 \mathrm{nl} / \mathrm{min}$. The needle was left in place for $5 \mathrm{~min}$ to ensure complete diffusion of the viruses and then slowly retracted from the brain. Both hemispheres were injected with the same shRNA.

\section{Accelerating rotarod}

Five weeks after intrastriatal injection of $\mathrm{rAAV} 2 / 8$ shRNAs, animals were subjected to the accelerating rotarod test to analyze motor learning $(n=8 \mathrm{WT}$ AAVshCtr, $n=8$ WT AAV-shRTP801, $n=9$ R6/1 AAV-shCtr, $n=9 \mathrm{R} 6 / 1$ AAV-shRTP801). Mice were placed on a $3 \mathrm{~cm}$ rod (Panlab) with an increasing speed from 4 to 40 r.p.m. over $5 \mathrm{~min}$. Latency to fall was recorded as the time mice spent in the rod before falling. Accelerating rotarod test was performed for 4 days, 3 trials per day. Trials in the same day were separated by $1 \mathrm{~h}$. Throughout experimentation and data analysis, the experimenter was blind to genotype and/or virus injected.

\section{Immunohistofluorescence of mouse brain sections}

Animals were deeply anesthetized with dolethal (Vétoquinol) $(60 \mathrm{mg} / \mathrm{kg})$, and intracardially perfused with $4 \%$ paraformaldehyde in phosphate-buffered saline (PBS) buffer $(\mathrm{pH}=7.2-7.4)$. Brains were removed and postfixed for $18-24 \mathrm{~h}$, and cryoprotected with $30 \%$ sucrose in PBS $0.02 \%$ sodium azide and frozen in dry-ice cooled 2methylbutane. Serial cryostat $25 \mu \mathrm{m}$-thick sections were collected in PBS $0.2 \%$ sodium azide as free-floating sections and processed for immunohistofluorescence. Sections were washed with PBS and incubated with $50 \mathrm{mM}$ $\mathrm{NH}_{4} \mathrm{Cl}$ for 30 min to block aldehyde free-induced fluorescence. Tissue was blocked with SuperBlock-PBS containing $0.3 \%$ Triton X-100 for $2 \mathrm{~h}$ at room temperature. After, slices were incubated overnight at $4{ }^{\circ} \mathrm{C}$ with the primary antibodies anti-GFP chicken polyclonal (Synaptic Systems, \#132006) diluted 1:500 in SuperBlock-PBS 0.3\% Triton X-100. Later, sections were washed in PBS and incubated for $2 \mathrm{~h}$ at room temperature with the secondary antibody goat anti-Chicken $\operatorname{IgY}(\mathrm{H}+\mathrm{L})$ conjugated to AlexaFluor $^{\text {TM }} 488$ (Thermo Fisher Scientific, \#A11039) diluted 1:500 in SuperBlock-PBS 0.3\% Triton X-100. For nuclear staining, nuclei were revealed with Hoechst 3342 diluted 1:5000 (Thermo Fisher Scientific, \#sc-H3570) along with the secondary antibody incubation. Slices were washed with PBS before being mounted with ProLong ${ }^{\mathrm{TM}}$ Gold Antifade Mountant on SuperFrost ${ }^{\mathrm{TM}}$ Plus Adhesion Slides (Thermo Fisher Scientific). Stained mouse brain sections were observed by epifluorescent microscopy.

\section{Experimental design and statistical analysis}

Sample sizes were calculated based on previous experiments. All experiments were performed at least in triplicate, and results are reported as mean \pm SEM. Normal distribution of data was tested using three different tests: D'Agostino-Pearson omnibus, Shapiro-Wilk and Kolmogorov-Smirnov tests. Normality was assumed when at least one of the tests was positive for normal distribution, and statistical analysis was performed using parametric statistical test. Otherwise non-parametric Mann-Whitney or Kruskal-Wallis or Dunn's test were performed. To compare variances $F$-test was performed. In experiments with normal distribution, Student's $t$-test was performed as unpaired, two-tailed sets of arrays and presented as probability $P$-values. One-way ANOVA with Bonferroni's or Dunnett's multiple comparison test and two-way ANOVA followed by Bonferroni's post hoc tests were performed for the comparison of multiple groups. All test performed are indicated in the figure legends. Grubbs' test was performed to determine the significant outlier values. Values of $P<0.05$ were considered as statistically significant. Statistical analyses were performed on GraphPath Prism. All experiments in this study were blinded and randomized. All mice bred for the experiments were used for preplanned experiments and randomized to experimental groups. Data were collected, processed, and analyzed randomly.

\section{Acknowledgements \\ We thank Dr. M MacDonald (Massachusetts General Hospital, Boston, Massachusetts, USA) for the Hdh ${ }^{\text {77/Q111 }}$ mice. The constructs Q25 Q72 and Q103 were a kind gift of Dr. G.M. Lawless (Cure HD Initiative, Reagent Resource Bank of the Hereditary Disease Foundation, New York, NY). We also thank the Neurological Tissue Bank of the Biobanc-Hospital Clínic-IDIBAPS (Barcelona, Spain) and Dr. Ellen Gelpi for providing human tissue samples, and Ana López for her technical support. We thank Maria Calvo from the Advanced Microscopy Unit, Scientific and Technological Centers, University of Barcelona, for their support and advice in confocal techniques. This work was funded by the Spanish Ministry of Economy and Competitivity with the following grants: SAF2017-8812-R(AEI/FEDER, UE) and SAF2014-57160-R(AEI/FEDER, UE).}

\section{Author details}

'Department of Biomedicine, Faculty of Medicine, University of Barcelona, 08036 Barcelona, Catalonia, Spain. ${ }^{2}$ Institut de Neurociències, University of Barcelona, 08036 Barcelona, Catalonia, Spain. ${ }^{3}$ IDIBAPS-Institut d'Investigacions Biomèdiques August Pi i Sunyer, 08036 Barcelona, Catalonia, Spain. ${ }^{4}$ Centro de 
Investigación Biomédica en Red sobre Enfermedades Neurodegenerativas (CIBERNED), 08036 Barcelona, Spain

\section{Data availability}

All data generated or analyzed during this study are included in this published article and the supplementary information files.

\section{Conflict of interest}

The authors declare that they have no conflict of interest.

\section{Ethical approval}

All procedures were performed in compliance with the NIH Guide for the Care and Use of Laboratory Animals and approved by the local animal care committee of Universitat de Barcelona following European (2010/63/UE) and Spanish (RD53/2013) regulations for the care and use of laboratory animals. Human samples were obtained following the guidelines and approval of the local ethics committee (Hospital Clínic of Barcelona's Clinical Research Ethics Committee).

\section{Publisher's note}

Springer Nature remains neutral with regard to jurisdictional claims in published maps and institutional affiliations.

Supplementary Information accompanies this paper at (https://doi.org/ 10.1038/s41419-020-02775-5).

Received: 8 May 2020 Revised: 8 July 2020 Accepted: 10 July 2020 Published online: 30 July 2020

\section{References}

1. Vonsattel, J. P. et al. Neuropathological classification of Huntington's disease. J. Neuropathol. Exp. Neurol. 44, 559-577 (1985).

2. Halliday, G. M. et al. Regional specificity of brain atrophy in Huntington's disease. Exp. Neurol. 154, 663-672 (1998).

3. MacDonald, M. E. et al. A novel gene containing a trinucleotide repeat that is expanded and unstable on Huntington's disease chromosomes. The Huntington's Disease Collaborative Research Group. Cell 72, 971-983 (1993).

4. Bañez-Coronel, M. et al. A pathogenic mechanism in Huntington's disease involves small CAG-repeated RNAs with neurotoxic activity. PLoS Genet. 8, e1002481 (2012).

5. Rosas, H. D. et al. Evidence for more widespread cerebral pathology in early HD: an MRI-based morphometric analysis. Neurology 60, 1615-1620 (2003).

6. Rüb, U. et al. Degeneration of the Cerebellum in Huntington's Disease (HD): possible relevance for the clinical picture and potential gateway to pathological mechanisms of the disease process. Brain Pathol. 23, 165-177 (2013).

7. Caramins, M., Halliday, G., McCusker, E. \& Trent, R. J. Genetically confirmed clinical Huntington's disease with no observable cell loss. J. Neurol. Neurosurg. Psychiatry 74, 968-970 (2003).

8. Levine, M. S., Cepeda, C., Hickey, M. A., Fleming, S. M. \& Chesselet, M.-F. Genetic mouse models of Huntington's and Parkinson's diseases: illuminating but imperfect. Trends Neurosci. 27, 691-697 (2004).

9. Turmaine, M. et al. Nonapoptotic neurodegeneration in a transgenic mouse model of Huntington's disease. Proc. Natl Acad. Sci. 97, 8093-8097 (2000).

10. DiFiglia, M. et al. Aggregation of huntingtin in neuronal intranuclear inclusions and dystrophic neurites in brain. Science 277, 1990-1993 (1997).

11. Cepeda, C. et al. NMDA receptor function in mouse models of Huntington disease. J. Neurosci. Res. 66, 525-539 (2001).

12. Hannan, A. J. Synaptopathy, circuitopathy and the computational biology of Huntington's disease. BMC Biol. 16, 71 (2018).

13. Costa-Mattioli, M., Sossin, W. S., Klann, E. \& Sonenberg, N. Translational control of long-lasting synaptic plasticity and memory. Neuron 61, 10-26 (2009).

14. Santini, E., Muller, R. U. \& Quirk, G. J. Consolidation of extinction learning involves transfer from NMDA-independent to NMDA-dependent memory. J. Neurosci. 21, 9009-9017 (2001).

15. Henry, F. E., Hockeimer, W., Chen, A., Mysore, S. P. \& Sutton, M. A. Mechanistic target of rapamycin is necessary for changes in dendritic spine morphology associated with long-term potentiation. Mol. Brain 10, 50 (2017).
16. Szabó, E. C., Manguinhas, R. \& Fonseca, R. The interplay between neuronal activity and actin dynamics mimic the setting of an LTD synaptic tag. Sci. Rep. 6, 33685 (2016).

17. Lipton, J. O. \& Sahin, M. The neurology of mTOR. Neuron 84, 275-291 (2014).

18. Sotrel, A., Williams, R. S., Kaufmann, W. E. \& Myers, R. H. Evidence for neuronal degeneration and dendritic plasticity in cortical pyramidal neurons of Huntington's disease: a quantitative Golgi study. Neurology 43, 2088-2096 (1993).

19. Usdin, M. T., Shelbourne, P. F., Myers, R. M. \& Madison, D. V. Impaired synaptic plasticity in mice carrying the Huntington's disease mutation. Hum. Mol. Genet. 8, 839-846 (1999).

20. Milnerwood, A. J. et al. Early development of aberrant synaptic plasticity in a mouse model of Huntington's disease. Hum. Mol. Genet. 15, 1690-1703 (2006).

21. Creus-Muncunill, J. et al. Increased translation as a novel pathogenic mechanism in Huntington's disease. Brain 142, 3158-3175 (2019).

22. Gines, S., Ivanova, E., Seong, I.-S., Saura, C. A. \& MacDonald, M. E. Enhanced Akt signaling is an early pro-survival response that reflects $\mathrm{N}$-methyl-D-aspartate receptor activation in Huntington's disease knock-in striatal cells. J. Biol. Chem. 278, 50514-50522 (2003).

23. Saavedra, $\mathrm{A}$ et al. $\mathrm{PH}$ domain leucine-rich repeat protein phosphatase 1 contributes to maintain the activation of the PI3K/Akt pro-survival pathway in Huntington's disease striatum. Cell Death Differ. 17, 324-335 (2010).

24. Creus-Muncunill, J. et al. Increased levels of rictor prevent mutant huntingtininduced neuronal degeneration. Mol. Neurobiol 55, 7728-7742 (2018).

25. Gao, T., Furnari, F. \& Newton, A. C. PHLPP: A phosphatase that directly dephosphorylates Akt, promotes apoptosis, and suppresses tumor growth. Mol. Cell 18, 13-24 (2005).

26. Bayascas, J. R. \& Alessi, D. R. Regulation of Akt/PKB Ser473 phosphorylation. Mol. Cell 18, 143-145 (2005).

27. Colin, E. et al. Akt is altered in an animal model of Huntington's disease and in patients. Eur. J. Neurosci. 21, 1478-1488 (2005).

28. Eldh, M. et al. Exosomes communicate protective messages during oxidative stress; possible role of exosomal shuttle RNA. PLoS ONE 5, e15353 (2010).

29. Martín-Flores, N. et al. RTP801 is involved in mutant huntingtin-induced cell death. Mol. Neurobiol. 53, 2857-2868 (2016).

30. Malagelada, C., Ryu, E. J., Biswas, S. C., Jackson-Lewis, V. \& Greene, L. A. RTP801 is elevated in Parkinson brain substantia nigral neurons and mediates death in cellular models of Parkinson's disease by a mechanism involving mammalian target of rapamycin inactivation. J. Neurosci. 26, 9996-10005 (2006).

31. Malagelada, C., Zong, H. J. \& Greene, L. A. RTP801 is induced in Parkinson's disease and mediates neuron death by inhibiting Akt phosphorylation/activation. J. Neurosci 28, 14363-14371 (2008).

32. Penrod, R. D., Campagna, J., Panneck, T., Preese, L. \& Lanier, L. M. The presence of cortical neurons in striatal-cortical co-cultures alters the effects of dopamine and BDNF on medium spiny neuron dendritic development. Front. Cell. Neurosci. 9, 269 (2015).

33. Penrod, R. D., Kourrich, S., Kearney, E., Thomas, M. J. \& Lanier, L. M. An embryonic culture system for the investigation of striatal medium spiny neuron dendritic spine development and plasticity. J. Neurosci. Methods 200, 1-13 (2011).

34. Fourie, C. et al. Differential changes in postsynaptic density proteins in postmortem Huntington's disease and Parkinson's disease human brains. J. Neurodegener. Dis. 2014, 1-14 (2014).

35. Pryor, W. M. et al. Huntingtin promotes mTORC1 signaling in the pathogenesis of Huntington's disease. Sci. Signal. 7, ra103-ra103 (2014).

36. Sarkar, S., Ravikumar, B., Floto, R. A. \& Rubinsztein, D. C. Rapamycin and mTORindependent autophagy inducers ameliorate toxicity of polyglutamineexpanded huntingtin and related proteinopathies. Cell Death Differ. 16, 46-56 (2009).

37. Ravikumar, B. et al. Inhibition of mTOR induces autophagy and reduces toxicity of polyglutamine expansions in fly and mouse models of Huntington disease. Nat. Genet 36, 585-595 (2004).

38. Mangiarini, L. et al. Exon I of the HD gene with an expanded CAG repeat is sufficient to cause a progressive neurological phenotype in transgenic mice. Cell 87, 493-506 (1996).

39. Wheeler, V. C. et al. Early phenotypes that presage late-onset neurodegenerative disease allow testing of modifiers in Hdh CAG knock-in mice. Hum. Mol. Genet. 11, 633-640 (2002).

40. Naver, B. et al. Molecular and behavioral analysis of the R6/1 Huntington's disease transgenic mouse. Neuroscience 122, 1049-1057 (2003). 
41. Giralt, A. et al. Increased PKA signaling disrupts recognition memory and spatial memory: role in Huntington's disease. Hum. Mol. Genet. 20, 4232-4247 (2011).

42. Brito, $V$. et al. Imbalance of $\mathrm{p} 75(\mathrm{NTR}) / \mathrm{TrkB}$ protein expression in Huntington's disease: implication for neuroprotective therapies. Cell Death Dis. 4, e595 (2013).

43. Suelves, N. et al. Early downregulation of p75NTR by genetic and pharmacological approaches delays the onset of motor deficits and striatal dysfunction in Huntington's disease mice. Mol. Neurobiol 56, 935-953 (2018).

44. Murphy, K. P. et al. Abnormal synaptic plasticity and impaired spatial cognition in mice transgenic for exon 1 of the human Huntington's disease mutation. J. Neurosci. 20, 5115-5123 (2000).

45. Cepeda, C. et al. Multiple sources of striatal inhibition are differentially affected in Huntington's disease mouse models. J. Neurosci. 33 , 7393-7406 (2013).

46. Richards, P. et al. Dendritic spine loss and neurodegeneration is rescued by Rab11 in models of Huntington's disease. Cell Death Differ. 18, 191-200 (2011).

47. Murmu, R. P., Li, W., Holtmaat, A. \& Li, J.-Y. Dendritic spine instability leads to progressive neocortical spine loss in a mouse model of Huntington's disease. J. Neurosci. 33, 12997-13009 (2013)

48. Ferrante, R. J., Kowall, N. W. \& Richardson, E. P. Proliferative and degenerative changes in striatal spiny neurons in Huntington's disease: a combined study using the section-Golgi method and calbindin D28k immunocytochemistry. J. Neurosci. 11, 3877-3887 (1991).

49. Graveland, G. A., Williams, R. S. \& DiFiglia, M. Evidence for degenerative and regenerative changes in neostriatal spiny neurons in Huntington's disease. Science 227, 770-773 (1985)

50. Kim, J., Bordiuk, O. L. \& Ferrante, R. J. Experimental models of HD and reflection on therapeutic strategies. Int. Rev. Neurobiol. 98, 419-481 (2011).

51. Puigdellívol, M. et al. A role for Kalirin-7 in corticostriatal synaptic dysfunction in Huntington's disease. Hum. Mol. Genet. 24, 7265-7285 (2015).

52. Martín-Aparicio, E. et al. Proteasomal-dependent aggregate reversal and absence of cell death in a conditional mouse model of Huntington's disease. J. Neurosci. 21, 8772-8781 (2001).

53. Díaz-Hernández, M. et al. Full motor recovery despite striatal neuron loss and formation of irreversible amyloid-like inclusions in a conditional mouse model of Huntington's disease. J. Neurosci. 25, 9773-9781 (2005).

54. Canals, J. M. et al. Brain-derived neurotrophic factor regulates the onset and severity of motor dysfunction associated with enkephalinergic neuronal degeneration in Huntington's disease. J. Neurosci. 24, 7727-7739 (2004).

55. Spires, T. L. et al. Dendritic spine pathology and deficits in experiencedependent dendritic plasticity in R6/1 Huntington's disease transgenic mice. Eur. J. Neurosci. 19, 2799-2807 (2004).

56. Suelves, N. et al. Early downregulation of p75NTR by genetic and pharmacological approaches delays the onset of motor deficits and striatal dysfunction in Huntington's disease mice. Mol. Neurobiol. 56, 935-953 (2019).

57. Heck, N., Betuing, S., Vanhoutte, P. \& Caboche, J. A deconvolution method to improve automated 3D-analysis of dendritic spines: application to a mouse model of Huntington's disease. Brain Struct. Funct. 217, 421-434 (2012).

58. Simmons, D. A. et al. A small molecule p75NTR ligand normalizes signalling and reduces Huntington's disease phenotypes in R6/2 and BACHD mice. Hum. Mol. Genet. 25, 4920-4938 (2016).

59. Xie, Y., Hayden, M. R. \& Xu, B. BDNF overexpression in the forebrain rescues Huntington's disease phenotypes in YAC128 mice. J. Neurosci. 30, 14708-14718 (2010).
60. Torres-Peraza, J. F. et al. Disruption of striatal glutamatergic transmission induced by mutant huntingtin involves remodeling of both postsynaptic density and NMDA receptor signaling. Neurobiol. Dis. 29, 409-421 (2008).

61. Anglada-Huguet, $M$. et al. Prostaglandin E2 EP2 activation reduces memory decline in R6/1 mouse model of Huntington's disease by the induction of BDNF-dependent synaptic plasticity. Neurobiol. Dis. 95 22-34 (2016).

62. Marco, S. et al. Suppressing aberrant GluN3A expression rescues synaptic and behavioral impairments in Huntington's disease models. Nat. Med. 19, 1030-1038 (2013).

63. Zhang, Z. et al. RTP801 is a critical factor in the neurodegeneration process of A53T a-synuclein in a mouse model of Parkinson's disease under chronic restraint stress. Br. J. Pharmacol. 175, 590-605 (2018).

64. Malagelada, C., Jin, Z. H., Jackson-Lewis, V., Przedborski, S. \& Greene, L. A. Rapamycin protects against neuron death in in vitro and in vivo models of Parkinson's disease. J. Neurosci. 30, 1166-1175 (2010).

65. Spinelli, M. et al. Brain insulin resistance impairs hippocampal synaptic plasticity and memory by increasing GluA1 palmitoylation through FoxO3a. Nat. Commun. 8, 2009 (2017)

66. Marino, S. et al. PTEN is essential for cell migration but not for fate determination and tumourigenesis in the cerebellum. Development 129, 3513-3522 (2002).

67. Park, J. et al. Combination therapy targeting Akt and mammalian target of rapamycin improves functional outcome after controlled cortical impact in mice. J. Cereb. Blood Flow. Metab. 32, 330-340 (2012).

68. Hoeffer, C. A. \& Klann, E. mTOR signaling: at the crossroads of plasticity, memory and disease. Trends Neurosci. 33, 67-75 (2010).

69. Sarbassov, D. D., Guertin, D. A., Ali, S. M. \& Sabatini, D. M. Phosphorylation and regulation of Akt/PKB by the Rictor-mTOR complex. Science (80-.). 307, 1098-1101 (2005).

70. Sommer, B., Köhler, M., Sprengel, R. \& Seeburg, P. H. RNA editing in brain controls a determinant of ion flow in glutamate-gated channels. Cell $\mathbf{6 7}$ 11-19 (1991).

71. Lu, W. et al. Subunit composition of synaptic AMPA receptors revealed by a single-cell genetic approach. Neuron 62, 254-268 (2009).

72. Man, H.-Y. GluA2-lacking, calcium-permeable AMPA receptors-inducers of plasticity? Curr. Opin. Neurobiol. 21, 291-298 (2011).

73. Reichardt, L. F. Neurotrophin-regulated signalling pathways. Philos. Trans. $R$. Soc. B Biol. Sci. 361, 1545-1564 (2006).

74. Chao, M. V. Neurotrophins and their receptors: a convergence point for many signalling pathways. Nat. Rev. Neurosci. 4, 299-309 (2003).

75. Kalb, R. The protean actions of neurotrophins and their receptors on the life and death of neurons. Trends Neurosci. 28, 5-11 (2005).

76. María Frade, J., Rodríguez-Tébar, A. \& Barde, Y.-A. Induction of cell death by endogenous nerve growth factor through its p75 receptor. Nature 383, 166-168 (1996).

77. Friedman, W. J. Neurotrophins induce death of hippocampal neurons via the p75 receptor. J. Neurosci. 20, 6340-6346 (2000).

78. Brito, V. et al. Neurotrophin receptor p75NTR mediates Huntington's diseaseassociated synaptic and memory dysfunction. J. Clin. Invest. 124, 4411-4428 (2014).

79. Canal, M. et al. Loss of NEDD4 contributes to RTP801 elevation and neuron toxicity: Implications for Parkinson's disease. Oncotarget 7, 58813-58831 (2016).

80. Romaní-Aumedes, J. et al. Parkin loss of function contributes to RTP801 elevation and neurodegeneration in Parkinson's disease. Cell Death Dis 5, e1364 (2014). 